\title{
Cu charge radii reveal a weak sub-shell effect at $N=40$
}

\author{
M. L. Bissell, ${ }^{1,2}$ T. Carette, ${ }^{3}$ K. T. Flanagan, ${ }^{1,4,2}$ P. Vingerhoets, ${ }^{1}$ J. Billowes, ${ }^{2}$ K. Blaum, ${ }^{5}$ B. Cheal,,${ }^{2,6}$ S. Fritzsche,, 8 \\ M. Godefroid, ${ }^{3}$ M. Kowalska, ${ }^{9}$ J. Krämer,,${ }^{10,11}$ R. Neugart, ${ }^{5,10}$ G. Neyens, ${ }^{1}$ W. Nörtershäuser, ${ }^{10,11}$ and D. T. Yordanov ${ }^{5,9,12}$ \\ ${ }^{1}$ Instituut voor Kern- en Stralingsfysica, KU Leuven, B 3001 Leuven, Belgium \\ ${ }^{2}$ School of Physics and Astronomy, The University of Manchester, Manchester M13 9PL, United Kingdom \\ ${ }^{3}$ Chimie quantique et photophysique, Université libre de Bruxelles, B 1050 Brussels, Belgium \\ ${ }^{4}$ I.P.N. Orsay, F-91940 Orsay Cedex, France \\ ${ }^{5}$ Max-Plank-Institut für Kernphysik, D-69117 Heidelberg, Germany \\ ${ }^{6}$ Oliver Lodge Laboratory, University of Liverpool, Liverpool L69 7ZE, United Kingdom \\ ${ }^{7}$ Helmholtz-Institut Jena, D-07743 Jena, Germany \\ ${ }^{8}$ Theoretisch-Physikalisches Institut, Friedrich-Schiller-Universität Jena, D 07743 Jena, Germany \\ ${ }^{9}$ Physics Department, CERN, CH-1211 Geneva 23, Switzerland \\ ${ }^{10}$ Institut für Kernchemie, Universität Mainz, D-55128 Mainz, Germany \\ ${ }^{11}$ Institut für Kernphysik, TU Darmstadt, D-64289 Darmstadt, Germany \\ ${ }^{12}$ CSNSM-IN2P3-CNRS, Université de Paris Sud, F-91405 Orsay, France
}

(Received 24 February 2016; published 17 June 2016)

\begin{abstract}
Collinear laser spectroscopy on ${ }^{58-75} \mathrm{Cu}$ isotopes was performed at the CERN-ISOLDE radioactive ion beam facility. In this paper we report on the isotope shifts obtained from these measurements. State-of-the-art atomic physics calculations have been undertaken in order to determine the changes in mean-square charge radii $\delta\left\langle r^{2}\right\rangle^{A, A^{\prime}}$ from the observed isotope shifts. A local minimum is observed in these radii differences at $N=40$, providing evidence for a weak $N=40$ sub-shell effect. However, comparison of $\delta\left\langle r^{2}\right\rangle^{A, A^{\prime}}$ with a droplet model prediction including static deformation deduced from the spectroscopic quadrupole moments, points to the persistence of correlations at $N=40$.
\end{abstract}

DOI: 10.1103/PhysRevC.93.064318

\section{INTRODUCTION}

Shell structure in atomic nuclei is known to evolve with $N$ and $Z$ and this has attracted significant attention in the last decades in different regions of the nuclear chart [1]. In the neutron rich nickel region (around $Z=28$ ) the $N=40$ harmonic oscillator magic number has been widely debated. The relatively high-lying first $I^{\pi}=2^{+}$state in ${ }^{68} \mathrm{Ni}$ suggested this nucleus to be doubly magic and thus $N=40$ to be a magic sub-shell gap [2]. Mass measurements are often considered as a test for shell and sub-shell closure. However, the nonobservation of a sub-shell effect at $N=40$ in the first mass measurements in this region [3] started the debate about whether or not $N=40$ is indeed a sub-shell gap. The low $B(E 2)$ transition rate in ${ }^{68} \mathrm{Ni}$, another signature of magicity [4], as well as the high $2^{+}$excitation energy were interpreted as to be mainly due to the parity-change between the $p f$ orbits below $N=40$ and the $g d s$ orbits above it [5]. More recent high-precision mass measurements from the CERN-ISOLTRAP and the IGISOL-JYFLTRAP Penning-trap experiments revealed evidence for only a very weak sub-shell closure at ${ }^{68} \mathrm{Ni}$, which is disappearing quickly when moving away from $Z=28[6,7]$.

To shed further light on the 'semimagic' behavior of $N=40$ isotopes around ${ }^{68} \mathrm{Ni}$, we investigated the ground-

Published by the American Physical Society under the terms of the Creative Commons Attribution 3.0 License. Further distribution of this work must maintain attribution to the author(s) and the published article's title, journal citation, and DOI. and isomeric-state properties of the $\mathrm{Cu}$ isotopes, having one proton outside the magic $Z=28$ shell gap. The magnetic dipole and electric quadrupole moments along the chain of Cu isotopes, from $N=29$ up to $N=46$, were measured through optical measurements using bunched beam collinear laser spectroscopy at CERN-ISOLDE [8-11]. The moments elucidate a 'magic' behavior around $N=40$ [9], but similar to the excitation energy and transition rates in $\mathrm{Ni}$ isotopes, this can be attributed to the parity-change of the neutron orbits, which prevent mixing of first-order $M 1$ and $E 2$ excitations into the ground state wave functions.

Although wide-scale theoretical reproduction of nuclear mean-square charge radii remains illusive, it is well known that this observable provides a highly sensitive test for nuclear shell effects. With only one notable exception for $N=20$ [12] a "kink" or local minimum is always found at shell closures [13] and is phenomenologically associated with a minimum in pairing and higher order correlations $[14,15]$ or deformation. Changes in the mean-square charge radius along a chain of isotopes can be deduced from the observed isotope shift in a given atomic transition $k$ with (transition) frequency $v_{k}$. The isotope shift depends on both the nuclear and electronic properties of the atom. Typically the observed IS between any pair of isotopes with masses $m_{A}$ and $m_{A^{\prime}}, \delta v_{k}^{A, A^{\prime}}$, is related to $\delta\left\langle r^{2}\right\rangle^{A, A^{\prime}}$, the change in mean-square charge radius between both isotopes using the following expression:

$\delta v_{k}^{A, A^{\prime}} \equiv v_{k}^{A^{\prime}}-v_{k}^{A}=M_{k}\left(\frac{m_{A^{\prime}}-m_{A}}{m_{A} m_{A^{\prime}}}\right)+F_{k} \delta\left\langle r^{2}\right\rangle^{A, A^{\prime}}$.

Here $M_{k}$ and $F_{k}$ are, respectively, the mass-shift and fieldshift parameters, both depending on the electronic transition. 
From this point the quantity $\delta\left\langle r^{2}\right\rangle$ will be used without superscripts where $A$ refers to our common reference isotope ${ }^{65} \mathrm{Cu}$.

Since the mean-square charge radius is known experimentally only for two $\mathrm{Cu}$ isotopes, accurate electronic structure calculations are required in order to deduce $\delta\left\langle r^{2}\right\rangle$ from the measured isotope shifts. To obtain these essential atomic parameters for $\mathrm{Cu}$, state-of-the-art multiconfiguration DiracHartree-Fock calculations have been performed, permitting a confident interpretation of the measured isotope shifts.

\section{EXPERIMENTS}

To produce the neutron-deficient $\mathrm{Cu}$ isotopes a $\mathrm{ZrO}_{2}$ target was bombarded with $1.4 \mathrm{GeV}$ protons at the CERNISOLDE radioactive ion beam facility, while the neutron-rich $\mathrm{Cu}$ isotopes have been produced using a $\mathrm{UC}_{x}$ target. The radioactive $\mathrm{Cu}$ isotopes were selectively ionized by the RILIS laser ion source [16]. These ions were accelerated to $30 \mathrm{keV}$ and mass separated with the high-resolution mass separator prior to injection into the gas-filled radiofrequency linear Paul trap ISCOOL [17]. Here the ions were accumulated for $50 \mathrm{~ms}$ and released as short bunches with a typical temporal width of $5 \mu \mathrm{s}$. The bunches of ions were then guided to the collinear laser spectroscopy setup where they were neutralized in a sodium-filled charge exchange cell (CEC). The fast atom beam emerging from the cell was overlapped in a collinear geometry with a $324.8 \mathrm{~nm}$ continuous-wave laser beam. Scanning across the $4 s^{2} S_{1 / 2}-4 p^{2} P_{3 / 2}^{o}$ atomic transition at $324.8 \mathrm{~nm}$ was

TABLE I. Isotope shifts and mean-square charge radii determined in this work with respect to ${ }^{65} \mathrm{Cu}$. Numbers in round brackets give the statistical uncertainty. The systematic error arising from uncertainty on the atomic factors $M_{k}$ and $F_{k}$ is contained within the square brackets.

\begin{tabular}{lccc}
\hline \hline$A^{\prime}$ & $I^{\pi}$ & $\delta v^{65, A^{\prime}}(\mathrm{MHz})$ & $\delta\left\langle r^{2}\right\rangle\left(\mathrm{fm}^{2}\right)$ \\
\hline 58 & $1^{+}$ & $-1975(10)$ & $-0.833(13)[91]$ \\
59 & $3 / 2^{-}$ & $-1717.4(70)$ & $-0.635(9)[71]$ \\
60 & $2^{+}$ & $-1415.0(60)$ & $-0.511(8)[57]$ \\
61 & $3 / 2^{-}$ & $-1147.9(50)$ & $-0.359(6)[40]$ \\
62 & $1^{+}$ & $-825.0(38)$ & $-0.293(5)[33]$ \\
63 & $3 / 2^{-}$ & $-576.1(11)$ & $-0.148(1)[17]$ \\
64 & $1^{+}$ & $-249.4(22)$ & $-0.116(3)[13]$ \\
65 & $3 / 2^{-}$ & 0 & 0 \\
66 & $1^{+}$ & $304.8(33)$ & $0.033(4)[12]$ \\
67 & $3 / 2^{-}$ & $561.4(35)$ & $0.115(5)[18]$ \\
68 & $1^{+}$ & $858.8(37)$ & $0.133(5)[31]$ \\
$68 m 1$ & $6^{-}$ & $812.5(26)$ & $0.192(3)[31]$ \\
69 & $3 / 2^{-}$ & $1079.0(20)$ & $0.238(3)[34]$ \\
70 & $6^{-}$ & $1347.3(23)$ & $0.271(3)[44]$ \\
$70 m 1$ & $3^{-}$ & $1334.6(84)$ & $0.287(11)[44]$ \\
$70 m 2$ & $1^{+}$ & $1307.1(83)$ & $0.323(11)[44]$ \\
71 & $3 / 2^{-}$ & $1526.5(91)$ & $0.407(11)[44]$ \\
72 & $2^{-}$ & $1787.1(38)$ & $0.429(5)[55]$ \\
73 & $3 / 2^{-}$ & $1984(12)$ & $0.523(15)[58]$ \\
74 & $2^{-}$ & $2260(14)$ & $0.505(18)[72]$ \\
75 & $5 / 2^{-}$ & $2484(16)$ & $0.546(21)[80]$ \\
\hline \hline
\end{tabular}

achieved by applying a voltage to the CEC in the $-10 \mathrm{kV}$ to $+10 \mathrm{kV}$ range. Spectra were recorded by monitoring the photon emission rate as a function of the applied voltage using two photomultiplier tubes (PMTs) located after the CEC. The background from scattered laser light and the PMT dark counts was suppressed by a factor of $10^{4}$ by only accepting counts when bunches of atoms passed in front of the PMTs. The hyperfine spectra for each isotope were subsequently fitted using a $\chi^{2}$ minimization procedure from which the hyperfine-structure centroids were obtained. More details on each experiment and the analysis procedure can be found in previous papers [8-11]. The energy of the beam from ISCOOL was determined by varying this parameter in order to reproduce the known ground state hyperfine splitting of ${ }^{65} \mathrm{Cu}$ [18]. From this procedure a total uncertainty on the beam energy of $\pm 7 \mathrm{~V}$ remained and the influence of this uncertainty on the isotope shifts and $\delta\left\langle r^{2}\right\rangle$ has been included in the statistical errors reported here. The isotope shifts obtained are presented in Table I.

\section{THEORETICAL ISOTOPE SHIFTS AND ELECTRONIC PARAMETERS}

We use the multiconfiguration Dirac-Hartree-Fock (MCDHF) method [19] for computing highly accurate wave functions for the $[\mathrm{Ar}] 3 d^{10} 4 s^{2} S_{1 / 2}$ and $[\mathrm{Ar}] 3 d^{10} 4 p^{2} P_{3 / 2}^{o}$ states of $\mathrm{Cu}$ I in order to estimate the $M_{k}$ and $F_{k}$ electronic parameters of Eq. (1). In the MCDHF theory, selected orbitals are optimized to find a minimum of a state-average configuration interaction energy functional. We also perform calculations without optimizing any orbital, but including nuclear finite mass and volume effects as well as quantum electrodynamic (QED) corrections. We refer to them as relativistic configuration interaction (RCI) calculations.

\section{A. Direct diagonalization of the Hamiltonian matrix}

One way to determine $M_{k}$ and $F_{k}$ using an ab initio method is to first compute the energies of the upper and lower atomic levels for several isotopes. This can be done by diagonalizing the full Hamiltonian matrix including the specific mass shifts (SMS) and the extended nuclear charge distribution [20,21]. For a given $\left(A, A^{\prime}, A^{\prime \prime}\right)$ triad, solving the $(2 \times 2)$ linear system of equations expressing the line shifts (1) for two isotope pairs in terms of $M_{k}$ and $F_{k}$ yields the two unknown electronic parameters $[22,23]$. This method has the advantage of providing a single set of mass- and field-shift parameters for a whole chain of isotopes, adopting the same standard parametrization as for the experimental analysis. It also allows the reliability of Eq. (1) to be estimated by investigating the magnitude of the variations in the calculated parameters for different choices of isotope triads. Its main disadvantage is that it requires the relativistic configuration interaction calculations to be performed for a series of selected isotopes. We first adopt this approach by computing the wave functions for the ${ }^{65} \mathrm{Cu}$ isotope that we use in RCI calculations for a series of five copper isotopes for the upper and lower levels (with mass numbers $A=59,61,63,65,66)$ to get the total energies and deduce the corresponding transition IS. The NMS and SMS 
TABLE II. Electronic mass and field-shift factors. $\bar{M}_{k}$ denotes calculations. See text for details on the different calculations and for the difference between $\bar{M}_{k}$ and $M_{k}$.

\begin{tabular}{lcccc}
\hline \hline & $\begin{array}{c}\Delta E \\
\left(\mathrm{~cm}^{-1}\right)\end{array}$ & $\begin{array}{c}F_{k} \\
\left(\mathrm{MHz} \mathrm{fm}^{-2}\right)\end{array}$ & $\begin{array}{c}\bar{M}_{k} \\
(\mathrm{GHz} \mathrm{u})\end{array}$ & $\begin{array}{c}M_{k} \\
(\mathrm{GHz} \mathrm{u})\end{array}$ \\
\hline $\mathrm{DF}$ & 25679 & -597 & 1166 & 1111 \\
LCM & 29244 & -680 & $1078^{\mathrm{a}}$ & \\
$\mathrm{CC}$ & 25744 & -662 & 924 & 862 \\
$\mathrm{CC}+\mathrm{CV}$ & 29892 & -767 & 1169 & 1103 \\
$\mathrm{CC}+\mathrm{CV}+\mathrm{ICV}$ & 29892 & -782 & 1156 & 1090 \\
MR-I RCI & 30748 & -779 & 1348 & 1283 \\
Extrapolated [36] & & & 1368 & 1303 \\
Experiment & 30784 & & & $1440(180)$ \\
Electron scattering & & & & $1413(27)$ \\
Muonic x-ray & & & & \\
\hline \hline
\end{tabular}

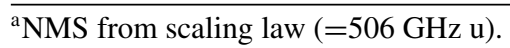

form together the total mass shift, $M_{k}=M_{k, \mathrm{NMS}}+M_{k, \mathrm{SMS}}$, as it occurs in Eq. (1). The NMS is estimated from the "scaling law" of the NMS approximation [24,25] using the observed transition frequency, $M_{k, \mathrm{NMS}}=\left(m_{e} / m_{n}\right) v_{k}$, while the SMS operator that is included in the Hamiltonian for the RCI calculations is limited to the standard mass polarization term $\left\langle\frac{1}{M} \sum_{i<j} \mathbf{p}_{i} \cdot \mathbf{p}_{j}\right\rangle$, as described in the write-up of the SMS92 computer program [26]. The results are reported in Table II. As far as the electron correlation models are concerned, we progressively improve the single-configuration Dirac-Fock (DF) approximation by incorporating single and double (SD) excitations from the $3 d$ and $4 s$ shells into one or two layers of correlation orbitals. In addition, we include single excitations from all occupied $1 s, \ldots, n l j$ sub-shells. We refer to these results as the "limited correlation model" (LCM), in contrast to the more elaborate models described below.

\section{B. Large scale multireference calculations and relativistic corrections to the recoil operator}

We carry out complementary large scale MCDHF calculations with the last version of the GRASP2K package [27] and computing $M_{k}$ and $F_{k}$ as expectation values of the relevant operators [28] using the ${ }^{65} \mathrm{Cu}$ wave function. In this scheme, $M_{k}$ is the difference in the expectation values between the two levels of the recoil Hamiltonian containing both the oneand two-body terms constituting, respectively, of the NMS and SMS, as described in Ref. [28]. In the case of $\mathrm{Cu} \mathrm{I,}$ $F_{k}$ can be reliably computed using the electron density at the nucleus $[29,30]$. A specificity of the present work is that we test the impact of relativistic corrections to the recoil operator as originally derived by Shabaev [31,32]. For this, we compare the results obtained with SMS92 [26] (denoted $\bar{M}_{k}$ ), based on the Dirac kinetic form of the NMS [25] and the mass polarization term $\left\langle\frac{1}{M} \sum_{i<j} \mathbf{p}_{i} \cdot \mathbf{p}_{j}\right\rangle$ for the SMS, with the values (denoted $M_{k}$ ) obtained with the more recent computer code RIS3 [28] using the relativistically corrected recoil operator [33]. Recalling that the two main configurations are of the type $[\mathrm{Ar}] 3 d^{10} n \ell$, with $(n \ell)=4 s / 4 p$, we will distinguish three types of double excitations: (i) core-core (CC), involving $n=2,3$ electrons; (ii) core-valence (CV), involving $n=3$ and
$4 s / 4 p$ electrons, and (iii) inner-core-valence (ICV), involving $1 s^{2}$ and $4 s / 4 p$ electrons. All calculations are performed with a common orbital basis for the $4 s$ and $4 p$ states, i.e., "Extended Optimized Level" (EOL) type of calculations [19] on the two states. The orbital active set (AS) is extended layer by layer, and only the last layer is optimized at each subsequent MCDHF calculation.

The configuration state functions (CSF) that are considered in the wave function expansions are limited to excitations of specific sub-shells. We first optimize the $n=5$ layer on core-core (CC) correlation. In a second series of calculations, we add core-valence $(\mathrm{CC}+\mathrm{CV})$ excitations, extending the AS until convergence, up to $n_{\max } l_{\max }=10 h$. In a third set of calculation, we add core-valence and inner-core-valence excitations $(\mathrm{CC}+\mathrm{CV}+\mathrm{ICV})$. For these, we reach a satisfactory convergence at $n_{\max } l_{\max }=12 \mathrm{~h}$. The numerical results are summarized in Table II for the different calculations. As it can be seen from this table, including single and double excitations of the main configuration recovers only $83 \%$ of the total electron correlation contribution $\left(5105 \mathrm{~cm}^{-1}\right)$ to the $\Delta E\left(4 s_{1 / 2}-4 p_{3 / 2}\right)$ transition energy.

An efficient way for capturing more electron correlation is to predefine a multireference (MR) space spanned by the most important configuration state functions giving a good zerothorder physical picture of the desired state. A multireference interacting (MR-I) expansion is then built [34], containing all CSFs that can be produced for a given orbital active set with the restriction that they interact to first order with at least one of the reference states of the MR space through the Dirac-Coulomb Hamiltonian [19]. Ab initio predictions can then be supported by a series of results obtained by extending systematically the orbital active set on the one hand, and the multireference space on the other hand. Using this approach, we merged the previous CSF lists with MR-I expansions built on extended MR spaces including the most important CSFs with one hole in the $3 d$ sub-shell. The resulting lists are used in multireference $\mathrm{RCI}$, including Breit interaction and vacuum-polarization. The results obtained with the largest calculations are reported in Table II under the label "MR-I RCI".

It has been observed that the experimental energy difference between the two atomic levels provides a good guideline for estimating mass isotope shifts [34,35]. When including MR-I sets in the calculations, transition energies above $30000 \mathrm{~cm}^{-1}$ are obtained, approaching the experimental value of $30784 \mathrm{~cm}^{-1}$, and a linear correlation can be observed between $\Delta E$ and $M_{k}$ for the different elaborate calculations, as illustrated in Ref. [36] and Fig. 1. This is consistent with previous observations [35,37]. It means that, within our model, the error on the computed $M$ values is correlated to the error on the transition energy. Hence we gain in precision by further extrapolating the computed results towards the experimental energy difference. From the observed convergence patterns, we deduce an intrinsic error of about $3-4 \%$ in the final extrapolated values.

A range of experimental $M_{k}$ values can be determined from electron scattering [38-40] and from muonic atom measurements $[41,42]$ once the field factor has been determined. These $M_{k}$ values have been obtained from Eq. (1) by combining our experimental $\delta \nu^{65,63}$ and the experimental $\delta\left\langle r^{2}\right\rangle^{65,63}$ with the 


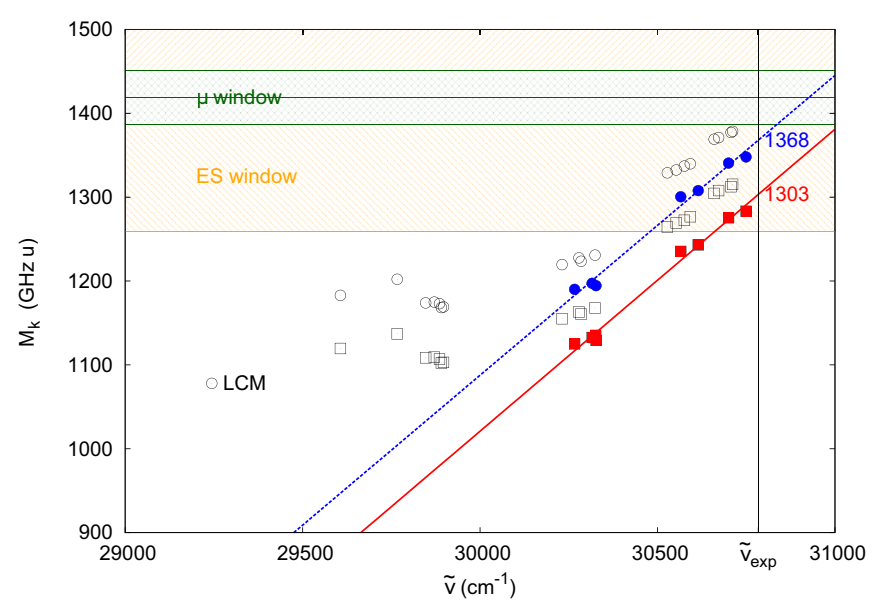

FIG. 1. Mass factor $(M)$ versus transition energy ( $\tilde{v})$ plot for RCI calculations with a gradually increasing number of correlations included. The $M$ values allowed by the muonic ( $\mu$ window) and electron scattering (ES window) measurements are shown in green and yellow, respectively. The vertical line indicates the experimental transition energy. Predicted $M$ values obtained with (squares, red) or without (circles, blue) relativistic corrections to the mass shift operators are deduced from extrapolation of results obtained with $n \geqslant 8$ to the experimental transition energy. LCM refers to the limited correlation model.

final $F_{k}=-779 \mathrm{MHz} \mathrm{fm}^{-2}$ theoretical value. As illustrated in Table II, the extrapolated $M_{k}$ values [36] show good agreement with the $M_{k}$ value based on electron scattering data, but are a few percent below the value from muonic atom data and not consistent within errors. Our methodology is unable to provide uncertainties related to types of correlation effects which are neglected. Assuming the correlation between $M_{k}$ and $v_{k}$ is similar when including neglected correlation effects we have a total additional uncertainty of $5 \%$. All in all, we see that it is necessary to assume a $5-10 \%$ uncertainty on the final extrapolated theoretical value of Table II. With this level of uncertainty the calculated $F_{k}$ and $M_{k}$ form a consistent set when the muonic atom $\delta\left\langle r^{2}\right\rangle^{65,63}$ and observed $\delta \nu^{65,63}$ are considered.

\section{CHANGES IN MEAN-SQUARE CHARGE RADII AND THE $N=40$ SUB-SHELL CLOSURE}

The calculated field and mass factors (the extrapolated values from Table II) were used to extract the mean-square charge radii from measured isotope shifts. If Eq. (1) is used, the values connected by a black dashed line in Fig. 2 are obtained. This trend obtained from the calculated $M_{k}$ value appears to be slightly flatter than the corresponding $\delta\left\langle r^{2}\right\rangle$ trends in the neighboring $\mathrm{Zn}$ and $\mathrm{Ni}$ isotopic chains. The full black line has been obtained with the calculated $F_{k}$ with a $10 \%$ uncertainty, and the corresponding $M_{k}$ value deduced from the muonic atom $\delta\left\langle r^{2}\right\rangle^{65,63}=0.148(17) \mathrm{fm}^{2}$. The result of this procedure yields the changes in mean-square charge radii presented in Table I. In Fig. 2 it can be seen that this procedure produces excellent consistency between the $\delta\left\langle r^{2}\right\rangle$ of $\mathrm{Cu}$ and those of its neighbors.

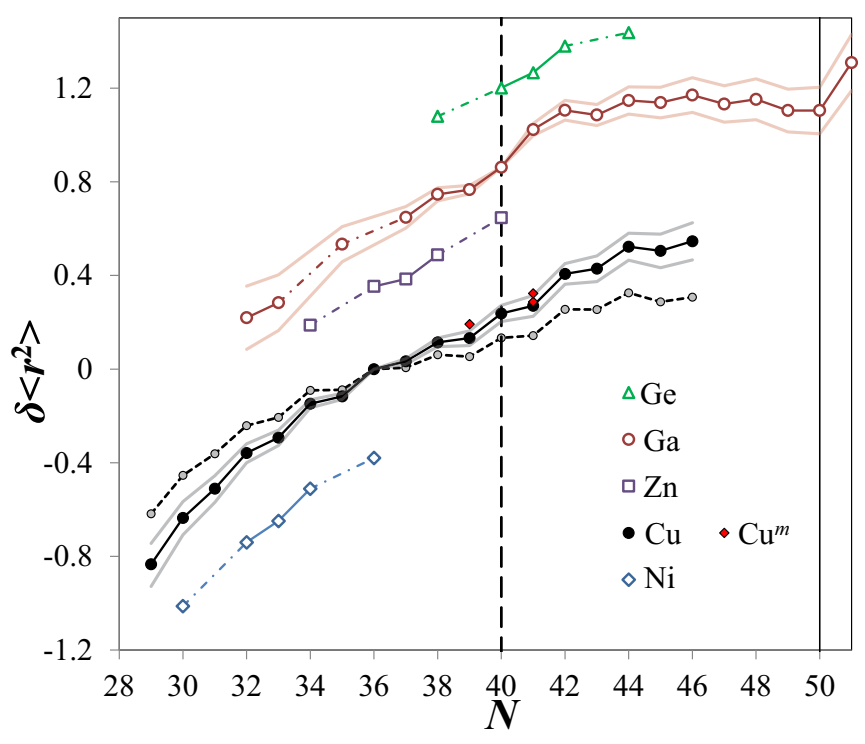

FIG. 2. Changes in mean-square charge radii for the $\mathrm{Cu}$ isotopes and neighboring isotopic chains. The other isotopic chains are offset by the nominal differences in absolute mean-square charge radii from [42] without further consideration of the associated large and model-dependent uncertainties. $\mathrm{Cu}$ results based only on the MCDHF calculations, without alteration of $M_{k}$ to reproduce the muonic atom data are shown as the black dashed line. Systematic uncertainties for $\mathrm{Cu}$ and $\mathrm{Ga}[43]$ are represented by the shaded lines above and below the respective trends.

Given that all isotopic chains contained within Fig. 2 were calibrated by, or directly obtained from, the same muonic atom data analysis, one cannot simply conclude that the few percent variation in the required mass shift factor is indeed due to a residual uncertainty in the MCDHF calculation. In addition to the untreated model uncertainties associated with the choice of charge distribution, the evaluation of muonic atom data also relies on a number of other nuclear observables and models for the calculation of polarization corrections [42]. Further confidence in the trends of charge radii within this region would require either additional electron scattering data and/or a critical reevaluation of muonic atom results.

In a first approximation, using the nuclear liquid drop model, mean-square charge radii are known to follow a smooth increasing trend with neutron number due to the increasing volume contribution. Deviations from this trend are indicative of correlations, or deformation.

The influence of deformation on $\left\langle r_{\mathrm{c}}^{2}\right\rangle$ is formally given by the expression

$$
\left\langle r_{\mathrm{c}}^{2}\right\rangle=\left\langle r_{\mathrm{c}}^{2}\right\rangle_{0}\left(1+\frac{5}{4 \pi} \sum_{i=2}^{\infty}\left\langle\beta_{i}^{2}\right\rangle\right),
$$

in which $\beta_{i}$ is the deformation parameter of order $i$, and $\left\langle r_{\mathrm{c}}^{2}\right\rangle_{0}$ is the mean-square charge radius of the spherical nucleus of equal volume. Clearly due to the dependence on $\left\langle\beta_{2}^{2}\right\rangle$ an increase in quadrupole deformation (either oblate or prolate) must always result in an increase in $\left\langle r_{\mathrm{c}}^{2}\right\rangle$.

In addition to the deformation of the nucleus, an increase in proton-pairing or higher-order correlations must always result 


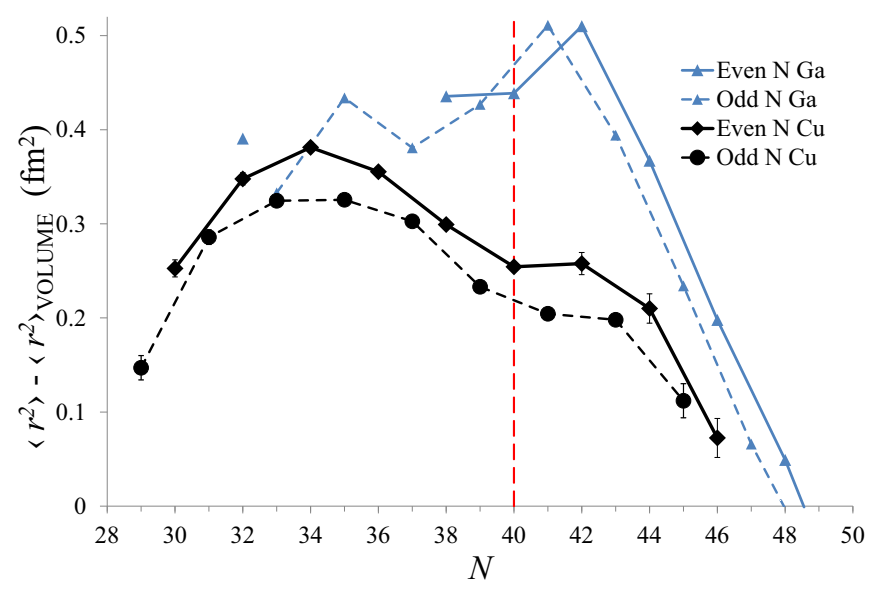

FIG. 3. Changes in mean-square charge radii for the $\mathrm{Cu}$ and $\mathrm{Ga}$ isotopes with a droplet model [44] volume contribution subtracted.

in an increase in charge radius, as long as the basis states employed consistently demonstrate larger $\left\langle r^{2}\right\rangle$ for lower binding energy. It should be emphasized here that such a mechanism strictly requires an increase in correlations within the proton sector. However, it has been demonstrated within a shellmodel framework that as multiparticle multihole correlations increase in the neutron sector proton correlations automatically increase [14]. Consequently, neutron shell closures not only correspond to a minimum in neutron correlations, but also a minimum in charge radii.

Both deformation and pairing or higher order correlations increase $\left\langle r_{\mathrm{c}}^{2}\right\rangle$ and it is for this reason that local minima are observed for closed shell nuclei. In order to obtain a clear view of these effects we subtract the droplet model predicted $\left\langle r^{2}\right\rangle$ for spherical nuclei [44] from the experimental $\left\langle r^{2}\right\rangle$ obtained by summing our $\delta\left\langle r^{2}\right\rangle$ values to $\left\langle r_{\mathrm{c}}^{2}\right\rangle$ of ${ }^{65} \mathrm{Cu}$ [42]. The results of this process are presented in Fig. 3 for the odd and even isotopes separately.

Apart from the expected parabolic behavior between the two well-established shell gaps at $N=28$ and towards $N=$ 50 , a weak influence of the $N=40$ sub-shell closure is apparent in the $\mathrm{Cu}$ chain. The characteristic parabolic trend for the odd and even isotopes is suppressed around $N=40$ by approximately the level of the normal isotopic odd-even staggering. Consequently it would appear that the $N=40$ sub-shell gap does indeed introduce a small reduction in correlations or deformation, however this reduction is similar in magnitude to the "blocking" effect of an odd neutron [45]. By comparison with the $\mathrm{Ga}$ isotopic chain $(Z=31)$ [43] it seems that the subtle influence of $N=40$ disappears quickly when moving away from $Z=28$. No clear minimum is seen at $N=40$, although the inversion of the normal odd-even staggering at $N=40$ hinders the direct interpretation of the observed radii differences. Note that an inversion in the odd-even staggering has also been observed in the light $\mathrm{Kr}$ $(Z=36)$ and $\operatorname{Sr}(Z=38)$ region below $N=45$ [46]. Here the inversion was attributed to the odd neutron polarizing the core into stable rigid deformation [47].

In order to assess how much of the residual $\delta\left\langle r^{2}\right\rangle$ presented in Fig. 3 can be ascribed to deformation of the charge

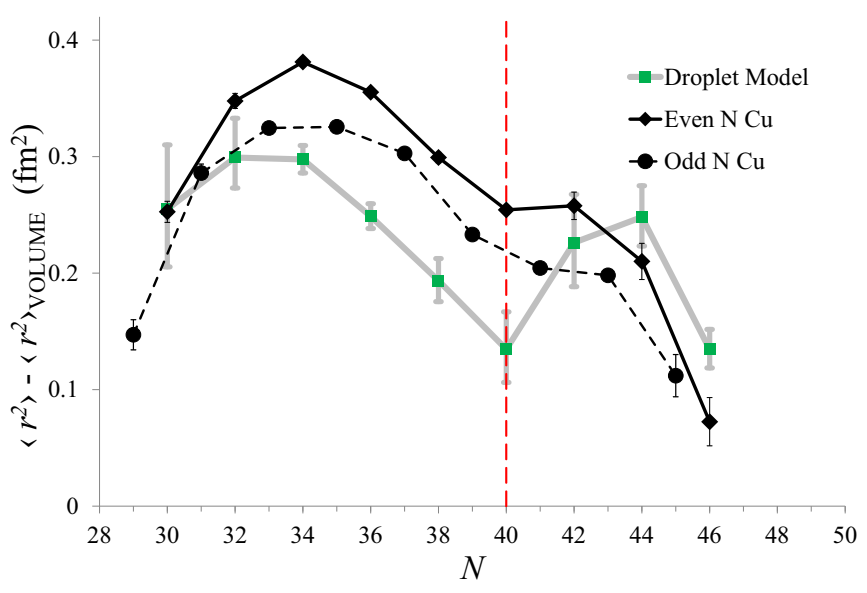

FIG. 4. Residual changes in mean-square charge radii for the $\mathrm{Cu}$ isotopes compared to droplet model [44] predictions based on experimental quadrupole moments.

distribution, one would require a good estimate of the mean square quadrupole moment in the intrinsic frame $\left\langle Q_{0}^{2}\right\rangle$ which would normally be associated with the $B(E 2)$ transition probabilities in even-even nuclei. As the $B(E 2)$ transition probabilities for the neighboring $\mathrm{Ni}$ and $\mathrm{Zn}$ isotopes do not simultaneously cover the full region of measured $\mathrm{Cu}$ isotopes, we proceed by estimating the deformation contribution using the spectroscopic quadrupole moments obtained from the observed hyperfine structures [11]. It should be noted that the quadrupole moment obtained in this way can only be related to $\left\langle Q_{0}\right\rangle^{2}$ and this value must always be less than or equal to $\left\langle Q_{0}^{2}\right\rangle$. Consequently when making the approximation $\left\langle Q_{0}^{2}\right\rangle=\left\langle Q_{0}\right\rangle^{2}$ in order to estimate $\left\langle r^{2}\right\rangle$ within a droplet-model framework, the resulting values should be considered a lower limit. In order to project the spectroscopic quadrupole moment onto the intrinsic frame the expression

$$
Q_{s}=\frac{3 K^{2}-I(I+1)}{(I+1)(2 I+3)} Q_{0}
$$

is used. Here, $K$ is the projection of the nuclear spin $I$ onto the deformation axis. For well-deformed ground state nuclei one would normally assume $K=I$. In the specific case of $\mathrm{Cu}$ with only one proton above the $N=28$ major shell closure, this assumption would not normally be considered safe. Fortunately for the $I=\frac{3}{2}$ even- $N$ isotopes, any possible assignment of $K$ results in the same $Q_{0}$ with only a sign change. Consequently for these isotopes the $\delta\left\langle r^{2}\right\rangle$ calculated from the spectroscopic quadrupole moment is to a significant extent independent of the value of $K$. For the odd-odd isotopes no such conclusion can be drawn, therefore we restrict further analysis to the even $N$ isotopes. In Fig. 4 the residual contributions to $\delta\left\langle r^{2}\right\rangle$ have been calculated from the measured quadrupole moments under the assumption of $K=I$. For ${ }^{75} \mathrm{Cu}$ with $I=\frac{5}{2}$, a wide range of values for $Q_{0}$ would also be obtained if other values for $K$ were considered. However only the assumption of $K=I$ appears to provide a consistent deformation for this isotope. Here it can be seen that the deformation corrected droplet model provides an excellent description of the observed $\delta\left\langle r^{2}\right\rangle$ values approaching 
$N=28$ and $N=50$. However the predicted radii significantly underestimate the observed charge radii in the midshell region. Specifically, if the experimental charge radii had been driven by changes in static deformation alone, the decrease at $N=40$ would have been much larger. From this it can only be concluded that pairing or higher order correlations develop in the midshell region and these correlations persist at $N=40$, leading to only a weak sub-shell closure effect in the charge radii of $\mathrm{Cu}$.

\section{SUMMARY}

Using collinear laser spectroscopy we have measured the optical isotope shifts for $17 \mathrm{Cu}$ isotopes. State-of-the art large-scale MCDHF calculations including different types of configuration mixing calculations yielded values for the mass shift and field shift in agreement with the muonic atomic data $\delta\left\langle r_{\mathrm{c}}^{2}\right\rangle^{65,63}$. In order to reproduce the overall trend in the charge radii in the trans-Ni region, we finally adopted the calculated field shift, but used the experimentally determined specific mass shift factor for extracting the change in mean-square charge radii from the measured isotope shifts. The charge radii obtained from these measurements, together with advanced atomic calculations, reveal a weak sub-shell effect at $N=40$ in the $\mathrm{Cu}$ isotopes having just one proton more than the magic $\mathrm{Ni}$ chain. It will be interesting to study in the future the charge radii of $\mathrm{Ni}$ isotopes in this neutron-rich region, to see if this weak sub-shell effect at $N=40$ is enhanced for the debated "doubly magic" ${ }^{68} \mathrm{Ni}$ isotope.

\section{ACKNOWLEDGMENTS}

This work has been supported by the German Ministry for Education and Research (BMBF) under Contract Nos. 05P12RDCIC and 05P15RDCIA, the UK Science and Technology Facilities Council (STFC), the FWO-Vlaanderen (Belgium), the EU Sixth Framework through No. Eurons506065, the BriX IAP Research Programs No. P6/23 and P7/12(Belgium), the Max-Planck Society. M.K. was supported by the EU (MEIF-CT-2006-042114). We thank the ISOLDE technical group for their support and assistance during this project.
[1] O. Sorlin and M.-G. Porque, Prog. Part. Nucl. Phys. 61, 602 (2008).

[2] R. Broda, B. Fornal, W. Królas, T. Pawłat, D. Bazzacco, S. Lunardi, C. Rossi-Alvarez, R. Menegazzo, G. de Angelis, P. Bednarczyk, J. Rico, D. De Acuña, P. J. Daly, R. H. Mayer, M. Sferrazza, H. Grawe, K. H. Maier, and R. Schubart, Phys. Rev. Lett. 74, 868 (1995).

[3] H. L. Seifert, J. M. Wouters, D. J. Vieira, H. Wollnik, X. G. Zhou, X. L. Tu, Z. Y. Zhou, and G. W. Butler, Z. Phys. A 349, 25 (1994).

[4] O. Sorlin et al., Phys. Rev. Lett. 88, 092501 (2002).

[5] K. Langanke, J. Terasaki, F. Nowacki, D. J. Dean, and W. Nazarewicz, Phys. Rev. C 67, 044314 (2003).

[6] S. Rahaman, J. Hakala, V. -V. Elomaa, T. Eronen, U. Hager, A. Jokinen, A. Kankainen, I. D. Moore, H. Penttil, S. Rinta-Antila, J. Rissanen, A. Saastamoinen, C. Weber, and J. Äystö, Eur. Phys. J. A 34, 5 (2007).

[7] C. Guénaut, G. Audi, D. Beck, K. Blaum, G. Bollen, P. Delahaye, F. Herfurth, A. Kellerbauer, H.-J. Kluge, J. Libert, D. Lunney, S. Schwarz, L. Schweikhard, and C. Yazidjian, Phys. Rev. C 75, 044303 (2007).

[8] K. T. Flanagan et al., Phys. Rev. Lett. 103, 142501 (2009).

[9] P. Vingerhoets, K. T. Flanagan, M. Avgoulea, J. Billowes, M. L. Bissell, K. Blaum, B. A. Brown, B. Cheal, M. De Rydt, D. H. Forest, C. Geppert, M. Honma, M. Kowalska, J. Krämer, A. Krieger, E. Mané, R. Neugart, G. Neyens, W. Nörtershäuser, T. Otsuka, M. Schug, H. H. Stroke, G. Tungate, and D. T. Yordanov, Phys. Rev. C 82, 064311 (2010).

[10] K. T. Flanagan, P. Vingerhoets, M. L. Bissell, K. Blaum, B. A. Brown, B. Cheal, M. De Rydt, D. H. Forest, C. Geppert, M. Honma, M. Kowalska, J. Krämer, A. Krieger, E. Mané, R. Neugart, G. Neyens, W. Nörtershäuser, M. Schug, H. H. Stroke, and D. T. Yordanov, Phys. Rev. C 82, 041302(R) (2010).

[11] P. Vingerhoets, K. Flanagan, J. Billowes, M. Bissell, K. Blaum, B. Cheal, M. D. Rydt, D. Forest, C. Geppert, M. Honma, M. Kowalska, J. Krämer, K. Kreim, A. Krieger, R. Neugart, G.
Neyens, W. Nörtershäuser, J. Papuga, T. Procter, M. Rajabali, R. Sánchez, H. Stroke, and D. Yordanov, Phys. Lett. B 703, 34 (2011).

[12] D. M. Rossi, K. Minamisono, H. B. Asberry, G. Bollen, B. A. Brown, K. Cooper, B. Isherwood, P. F. Mantica, A. Miller, D. J. Morrissey, R. Ringle, J. A. Rodriguez, C. A. Ryder, A. Smith, R. Strum, and C. Sumithrarachchi, Phys. Rev. C 92, 014305 (2015).

[13] I. Angeli and K. Marinova, At. Data Nucl. Data Tables 99, 69 (2013).

[14] E. Caurier, K. Langanke, G. Martnez-Pinedo, F. Nowacki, and P. Vogel, Phys. Lett. B 522, 240 (2001).

[15] G. A. Lalazissis and S. E. Massen, Phys. Rev. C 55, 2427 (1997)

[16] U. Köster et al., Nucl. Instrum. Methods Phys. Res. B 160, 528 (2000).

[17] H. Franberg et al., Nucl. Instrum. Methods Phys. Res. B 266, 4502 (2008).

[18] Y. Ting and H. Lew, Phys. Rev. 105, 581 (1957).

[19] I. P. Grant, Relativistic Quantum Theory of Atoms and Molecules: Theory and Computation - Atomic, Optical and Plasma Physics (Springer, New York, 2007).

[20] F. Parpia, C. F. Fischer, and I. Grant, Comput. Phys. Commun. 94, 249 (1996).

[21] S. Fritzsche, J. Electron Spectrosc. Relat. Phenom. (Proc. Eight Intl. Conf. Electronic Spectrosc. Struct.) 114-116, 1155 (2001).

[22] S. Fritzsche, Comput. Phys. Commun. 183, 1525 (2012).

[23] B. Cheal, T. E. Cocolios, and S. Fritzsche, Phys. Rev. A 86, 042501 (2012).

[24] C. W. P. Palmer, J. Phys. B 20, 5987 (1987).

[25] J. G. Li, C. Nazé, M. Godefroid, G. Gaigalas, and P. Jönsson, Eur. Phys. J. D 66, 290 (2012).

[26] P. Jönsson and C. F. Fischer, Comput. Phys. Commun. 100, 81 (1997).

[27] P. Jönsson, G. Gaigalas, J. Bieroń, C. F. Fischer, and I. Grant, Comput. Phys. Commun. 184, 2197 (2013). 
[28] C. Nazé, E. Gaidamauskas, G. Gaigalas, M. Godefroid, and P. Jönsson, Comput. Phys. Commun. 184, 2187 (2013).

[29] J. Ekman, private communication (2014).

[30] J. Li, C. Nazé, M. Godefroid, S. Fritzsche, G. Gaigalas, P. Indelicato, and P. Jönsson, Phys. Rev. A 86, 022518 (2012).

[31] V. M. Shabaev, Theor. Math. Phys. 63, 588 (1985).

[32] V. M. Shabaev, Sov. J. Nucl. Phys. 47(1), 69 (1988).

[33] E. Gaidamauskas, C. Nazé, P. Rynkun, G. Gaigalas, P. Jönsson, and M. Godefroid, J. Phys. B 44, 175003 (2011).

[34] T. Carette, C. Drag, O. Scharf, C. Blondel, C. Delsart, C. Froese Fischer, and M. Godefroid, Phys. Rev. A 81, 042522 (2010).

[35] T. Carette and M. R. Godefroid, Phys. Rev. A 83, 062505 (2011).

[36] T. Carette and M. R. Godefroid, arXiv:1602.06574 (2016).

[37] T. Carette and M. R. Godefroid, J. Phys. B 46, 095003 (2013).

[38] O. Schwentker, Ph.D. thesis, Institut für Kernphysik, Universitat Mainz, Germany (1977).

[39] W. Kegel, Master thesis, University of Amsterdam, The Netherlands (1977).
[40] N. G. Shevchenko, A. A. Khomich, A. Y. Buki, V. N. Polishchuk, B. V. Mazan'ko, and Y. A. Kasatkin, Yad. Fiz. 27, 731 (1978) [Sov. J. Nucl. Phys. 27, 159 (1978)].

[41] E. B. Shera, E. T. Ritter, R. B. Perkins, G. A. Rinker, L. K. Wagner, H. D. Wohlfahrt, G. Fricke, and R. M. Steffen, Phys. Rev. C 14, 731 (1976).

[42] G. Fricke and K. Heilig, Nuclear Charge Radii, LandoltBörnstein-Group I Elementary Particles, Nuclei and Atoms (Springer, Berlin/Heidelberg, 2004), Vol. 20.

[43] T. J. Procter et al., Phys. Rev. C 86, 034329 (2012).

[44] D. Berdichevsky and F. Tondeur, Z. Phys. A 322, 141 (1985).

[45] M. Horoi, Phys. Rev. C 50, 2834 (1994).

[46] M. Keim, E. Arnold, W. Borchers, U. Georg, A. Klein, R. Neugart, L. Vermeeren, R. Silverans, and P. Lievens, Nucl. Phys. A 586, 219 (1995).

[47] P. Lievens, E. Arnold, W. Borchers, U. Georg, M. Keim, A. Klein, R. Neugart, L. Vermeeren, and R. E. Silverans, Europhys. Lett. 33, 11 (1996). 